\title{
Factores edafoclimáticos en la producción y calidad del pasto Saboya (Holcus lanatus L.) en el Altiplano de Nariño
}

\author{
Edaphoclimatic factors in the production and quality of Saboya grass (Holcus lanatus L.) \\ in the highland of Nariño
}

Edmundo Apráez; ; Arturo Gálvez²; Julian Apráez³.

1 Zootecnista. MSc. Ph.D. Universidad de Nariño. San Juan de Pasto, Colombia, eapraez@gmail.com.

2 Zootecnista. MSc. Ph.D. Universidad de Nariño. San Juan de Pasto, Colombia, galvezceron@udenar.edu.co.

3 Ingeniero Agrónomo. MSc. Universidad de Nariño. San Juan de Pasto, Colombia, apraez.julian@gmail.com.

Citar: Apráez, E., Gálvez, A. \& Apráez, J. (2019). Factores edafoclimáticos en la producción y calidad del pasto Saboya (Holcus lanatus L.) en el Altiplano de Nariño. Revista de Ciencias Agrícolas. 36(1): 16-32. doi: http:// dx.doi.org/10.22267/rcia.193601.95

Recibido: Octubre $072016 . \quad$ Aceptado: febrero 07 de 2019.

\begin{abstract}
RESUMEN
La ganadería de leche en el Departamento de Nariño depende en gran medida de la oferta forrajera cuya calidad nutritiva y producción se ven afectadas principalmente por las condiciones climáticas. Para establecer su incidencia, se realizó la siguiente investigación en tres localidades del Municipio de Guachucal, entre 3050-3300msnm (zona 1); tres del Municipio de Ipiales, entre 2800-3049 msnm (zona 2) y tres del Municipio de Pasto, entre 2500-2650 msnm (zona 3). Se seleccionaron parcelas con pasto Saboya (Holcus lanatus L.) para el muestreo, sin actividades de laboreo, en las que se determinó variables climáticas, agronómicas, edáficas y bromatológicas, con la finalidad de establecer el grado de correlación entre ellas. Los resultados cuantitativos obtenidos se sometieron a un Análisis Multivariado de Componentes Principales (ACP) y los cualitativos a un Análisis de Correspondencias Múltiples (ACM). Se concluyó que para obtener los mejores rendimientos del pasto Saboya (H. lanatus) a alturas entre los 2800-3049 msnm, se necesita de un suelo suelto, materia orgánica de 5-6\% y un pH de 6 a 7 . Además, se determinó que $H$. lanatus crece bien a una temperatura de $10^{\circ} \mathrm{C}$, humedad relativa del $83 \%$, precipitación anual de 800 a 900mm y una luminosidad de 25klux. Entre 3050-3300 msnm, se requiere
\end{abstract}


de un suelo suelto, materia orgánica superior al 10\% y pH entre 5 y 6 . El pasto se adapta bien a una temperatura de 10 a $12^{\circ} \mathrm{C}$, humedad relativa del 86,28\% y precipitación pluvial de $923 \mathrm{~mm}$ anuales.

Palabras clave: pastos naturales, bromatológico, condiciones edáficas, parcela.

\begin{abstract}
The dairy farming in the Department of Nariño, depends mainly on the availability of forage, where its nutritional quality and production are affected by the climatic conditions. To establish its incidence, the research was carried out in three towns of the municipality of Guachucal, between 3050-3300 m.a.s.l. (zone 1); three of Municipality of Ipiales, between 2800-3049 m.a.s.l. (zone 2); and three of Municipality of Pasto, between 2500-2650 m.a.s.l. (zone 3). We selected plots with Saboya grass (Holcus lanatus L.) for sampling without activities of tillage, in which climatic, agronomic, edaphic and bromatological variables were determined, in order to stablish the degree of correlation between them. The quantitative variables analyzed under a Multivariate Analysis of Principal Components (APC), and Analyzes of Multiple Correspondences (AMC). It was concluded that the best yields of Saboya grass (H. lanatus), at a height between the 2800 - 3049 meters above sea level, need loose soils, organic matter of 5-6\% and a pH around 6 to 7. In addition, it grows well at a temperature of $10^{\circ} \mathrm{C}, 83 \%$ of relative humidity, 800 to $900 \mathrm{~mm}$ of annual rainfall and 25 klux of luminosity. In the range of height of 30503300 meters above sea level, is required of loose soils, organic matter superior to $10 \%$ and $\mathrm{pH}$ between 5 and 6 . The Saboya grass was adapted well to a temperature of 10 to $12^{\circ} \mathrm{C}, 86,28 \%$ of relative humidity and rainfall of $923 \mathrm{~mm}$ per year.
\end{abstract}

Keywords: bromatologic, soil conditions plot, natural grasses.

\section{INTRODUCCIÓN}

La ganadería nariñense se ha desarrollado siguiendo modelos tecnológicos foráneos que, si bien han dado buenos resultados productivos, en la práctica incurren en un incremento en los costos de producción y fundamentalmente en un impacto negativo sobre el ambiente al utilizar gran cantidad de insumos para la producción de forrajes para la alimentación del ganado. En el año 2004, el área cubierta por pastos y otras especies dedicadas a la ganadería era cercana a las 500 mil hectáreas (ENA-DANE, 2017), de las cuales, en ganadería especializada, la mayoría son manejadas con paquetes tecnológicos foráneos, que no han logrado adoptarse a condiciones tropicales, con un deterioro del terreno y una alta dependencia de recursos externos.

Esto pese a que, desde siempre, se ha contado con distintos recursos forrajeros en la zona, como pastos naturales y naturalizados, con los cuales se ha proporcionado alimento a los distintos animales de forma constante, a través de todo el año; no obstante, el afán de introducir especies que supuestamente brindan mejores resultados en el comportamiento productivo, ha desplazado a especies como la Saboya (H. lanatus L.) hasta el punto de relegarla $\mathrm{y}$ 
catalogarla como maleza, lo cual ha influido en la escasa investigación sobre sus condiciones de desarrollo, bondades agronómicas y nutritivas, Bernal (2003). Con el estudio de los factores que intervienen en el óptimo desarrollo, productividad y calidad de este pasto, es posible lograr mejores rendimientos e incrementar su valor nutricional, con el fin de establecer un modelo de producción sostenible, donde se manifieste una reducción de costos y un trabajo armónico con el entorno.

Para Begon et al. (1999), esta especie ha demostrado poseer una amplia adaptación a diversas condiciones de clima y suelo, llegando a producciones de materia seca y composiciones nutritivas interesantes desde el punto de vista económico, sobre todo en zonas marginales, donde otras especies de mayor producción necesitarían un mayor uso de insumos, o bien donde especies más exigentes en cuanto a condiciones químicas, físicas e hídricas del suelo no podrían desarrollarse. La presencia de $H$. lanatus en zonas agroecológicas diferentes se debe probablemente a la presencia de una alta variabilidad fenotípica, donde se distinguen ecotipos resultantes de un proceso deadaptación a condiciones ambientales determinadas, como la alta saturación de aluminio, bajo $\mathrm{pH}$, baja disponibilidad de fósforo, exceso de humedad en épocas lluviosas y periodos de sequía prolongados.

Bajo las anteriores consideraciones, esta investigación se propuso determinar los factores climáticos (temperatura, humedad relativa, luminosidad, precipitación y altitud) y de características del suelo (químicas, físicas y biológicas) que influyen en la producción y calidad nutricional del pasto Saboya en condiciones de no intervención, en la zona Andina de Nariño.

\section{MATERIALES Y MÉTODOS}

El estudio se realizó en nueve localidades, tres pertenecientes al Municipio de Guachucal (Zona 1), situadas entre 3050-3300 msnm; tres corresponden al Municipio de Ipiales (Zona 2), ubicadas entre 2800-3049 msnm y las tres restantes en el Municipio de Pasto (Zona 3), ubicadas entre 2500-2650 msnm (Cabrera, 2007).

En cada una se ubicaron tres áreas homogéneas (repeticiones) de praderas de Saboya (H. lanatus), en óptimas condiciones de producción, sin ningún tipo de intervención; de las cuales se tomó una muestra de pasto por réplica.

Variables climáticas. Las mediciones de temperatura y humedad relativa se hicieron con un higrotermógrafo LCD DIGTAL 2,8 EX modelo KTC-908, NEWEST IND00R/OUTD00R THERMOMETER-HUMIDITY marca ZEN; altura con un altímetro y la luminosidad con un luxómetro LIGHT METER WIDE RANGE EASYVIEW@ marca EXTECH INSTRUMENTS modelo EA90. Para la obtención de estos datos y los de precipitación, se recurrió a los reportes históricos del IDEAM desde el año 2002 hasta el 2008 (según el informe del IDEAM para el año 2017), realizando un promedio anual de cada una de las variables para su análisis.

Variables agronómicas. Entre las cuales están las siguientes:

Estado óptimo del pasto: basados en coloración verde oscura, cuya floración esté en capuchón, con el fin de asegurar su valor nutritivo, donde no haya sido intervenido por el hombre. 
Producción de forraje verde y materia seca: Se realizó un primer corte de pasto de 10 a $15 \mathrm{~cm}$ del suelo por réplica, para determinarlas, según la metodología propuesta por Apráez (1994). Con el segundo y tercer corte, realizados antes de cada floración, se precisó el periodo de recuperación del pasto.

Variables bromatológicas. Las diferentes muestras de pasto se analizaron en los laboratorios de Nutrición Animal de la Universidad de Nariño. Se evaluó la materia seca que se obtuvo mediante el método de secado en estufa, el nitrógeno total por la técnica Kjeldahl, Fibra Detergente Neutro (FDN), Fibra Detergente Acida (FDA), hemicelulosa, celulosa y lignina por Van Soest, minerales: Ca, P, Mg y S, de acuerdo con los procedimientos descritos por AOAC (Asociation Official Analitical Chemists, 1995).

Variables edáficas. Se analizaron de acuerdo con los procedimientos descritos por el Manual de Métodos Químicos para el Análisis de Suelos (Laboratorios de Análisis Especializados, UDENAR), así:

Variables químicas: Se analizó pH por el método potenciométrico, capacidad de intercambio cationico (CIC) mediante determinación por acetato de amonio, fósforo disponible a través del método Bray y Kurtz, materia orgánica por Walkey y Black, potasio de cambio, Mg, Fe, Zn y Ca determinados por absorción atómica, azufre mediante turbidimétria y Boro por determinación calorimétrica (Burbano, 2014).

Variables físicas: Se determinó la textura mediante el método del hidrómetro, densidad aparente por método de la probeta, densidad real a través del picnómetro, capacidad de campo por el método de columna de Chapingo, la porosidad total se calculó con base en las densidades real y aparente, la penetrabilidad se determinó con el penetrómetro, la infiltración fue evaluada a través del método de los cilindros concéntricos (Heredia, 2015).

Los datos registrados de las variables evaluadas fueron analizadas así: las variables cuantitativas que presentaron un coeficiente de variación (CV) mayor al 20\% se procesaron mediante el análisis de Componentes Principales (ACP) y las variables cualitativas se sometieron al análisis de Correspondencia Múltiple (ACM). El proceso se hizo mediante la utilización de software SPSS 15 y SAS versión 6 , respectivamente.

\section{RESULTADOS Y DISCUSIÓN}

Variables climáticas. Las determinaciones presentaron rangos muy estrechos entre las localidades de la Zona 1, obteniéndose un rango de temperatura de $8-10^{\circ} \mathrm{C}$, precipitación de 923,34 mm/anuales, luminosidad de 89,7 92,73 Klux y una humedad relativa de $86,28 \%$. Las veredas de la Zona 2 se caracterizaron por presentar una temperatura promedio de $10^{\circ} \mathrm{C}$, una precipitación anual promedio de $911,4 \mathrm{~mm} /$ anual, luminosidad de 25 Klux y 83\% humedad relativa. La zona 3 presentó una temperatura de $9,66^{\circ} \mathrm{C}$, precipitación de 1048,3 mm/año, luminosidad de 15 Klux y $79,6 \%$ humedad relativa (Tabla 1 y 2 ). 
Tabla 1. Condiciones óptimas para el desarrollo del pasto Saboya y actividades para situaciones específicas en las tres zonas.

\begin{tabular}{clll}
\hline Condición & \multicolumn{1}{c}{ Zona 1 } & \multicolumn{1}{c}{ Zona 2 } & \multicolumn{1}{c}{ Zona 3 } \\
\hline Tipo de suelo & Franco - arcilloso & Franco - arenoso & Franco \\
\% Materia orgánica & $13,52 \%$ & $8,4 \%$ & $8,07 \%$ \\
$\mathrm{pH}$ & 5.34 & 5,6 & 5,25 \\
To & $8-10^{\circ} \mathrm{C}$ & $10^{\circ} \mathrm{C}$ & $9,66^{\circ} \mathrm{C}$ \\
Penetrabilidad & $1,08 \mathrm{Mpa} / \mathrm{cm}^{2}$ & $1,8 \mathrm{Mpa} / \mathrm{cm}^{2}$ & $17,66 \mathrm{Mpa} / \mathrm{cm}^{2}$ \\
Luminosidad & $89,7-92,73 \mathrm{Klux}$ & $25 \mathrm{klux}$ & $15 \mathrm{Klux}$ \\
\hline Situación & \multicolumn{3}{c}{ Actividad } \\
Bajo contenido de & Es conveniente aportar materia orgánica, indispensable para el crecimiento y \\
materia orgánica & desarrollo del pasto. & \\
Mantenimiento de la & Realizar prácticas de agricultura de conservación (rotación de cultivos, \\
textura del suelo & labranza mínima y utilización de abonos verdes) que mejoran la infiltración \\
pH ácido & y la aireación. & \\
Porcentaje de & Incrementarlo incorporando caliza molida, CaCo3 y CaO. \\
porosidad bajo & Se debe aumentar la actividad biológica, sobre todo la subclase Oligochaeta, \\
& para que sean ellas las encargadas de cavar túneles y ayudar a mejorar la \\
\hline
\end{tabular}

Tabla 2. Rangos climáticos en las diferentes zonas de estudio

\begin{tabular}{clccccc}
\hline \multicolumn{2}{c}{ Localidad } & $\begin{array}{c}\text { Altitud } \\
\text { (msnm) }\end{array}$ & $\mathbf{T}^{\mathbf{0}}$ promedio & $\begin{array}{c}\text { P. pluvial } \\
\text { (mm/año) }\end{array}$ & $\begin{array}{c}\text { Humedad } \\
\text { relativa }\end{array}$ & $\begin{array}{c}\text { Luminosidad } \\
\text { (Klux) }\end{array}$ \\
\hline \multirow{2}{*}{ GUACHUCAL } & Arvela & 3094 & 10 & 923,34 & $86,28 \%$ & 94,71 \\
(zona 1) & Cualapud & 3244 & 8 & 923,34 & $86,28 \%$ & 92,73 \\
& Sta. Rosa & 3113 & 10 & 923,34 & $86,28 \%$ & 89,07 \\
\hline Promedio & & 3150,33 & 9,33 & 923,34 & 0,86 & 92,17 \\
\hline \multirow{2}{*}{ IPIALES } & Los marcos & 2850 & 10,8 & 917,7 & $84 \%$ & 29 \\
(zona 2) & San Luís & 2911 & 10 & $500-1000$ & $82,50 \%$ & 25 \\
& Sta. Bárbara & 3040 & 10 & $500-1000$ & $83 \%$ & 22 \\
\hline Promedio & & 2933,67 & 10,27 & 917,70 & 0,83 & 25,33 \\
\hline \multirow{2}{*}{$\begin{array}{c}\text { PASTO } \\
\text { (zona 3) }\end{array}$} & Mocondino & 2800 & 9 & 1300 & $77 \%$ & - \\
& S. Fernando & 2820 & 8 & 844,9 & $79 \%$ & - \\
\hline Promedio & Pasto & 2550,00 & 12,00 & $800-1000$ & 0,83 & - \\
\hline
\end{tabular}

Variables agronómicas. La producción de forraje verde $(\mathrm{FV})$ y periodo de recuperación promedio para la zona 1 fue $24.47 \mathrm{t} \mathrm{FV} /$ ha en 51 días, en la Zona 2 fue de $13 \mathrm{t} \mathrm{FV/ha} \mathrm{en} 40$ días, y para la zona 3 de $17.97 \mathrm{t} \mathrm{FV/ha} \mathrm{en} 46$ días. Como se observa en la Tabla 4, el rango óptimo para la recuperación del pasto Saboya, hasta prefloración, es de 30 - 45 días, debido la precocidad de su floración (Horwitz, 2005), por lo que se puede decir que el periodo de recuperación de las zonas 2 y 3 se ubican dentro de este rango, mientras que en la zona 1 el 
periodo de recuperación se extiende por fuera de él, situación causada por la diferencia de altitud entre las zonas. En cuanto a la producción de biomasa, se puede decir que, si bien los valores son superiores en la zona 1 , los de las zonas 2 y 3 no son nada despreciables, ya que la producción media de este forraje está en el rango de 12-15t $\mathrm{FV} /$ ha (Bernal 2003), como se muestra en la Tabla 3.

Tabla 3. Variables agronómicas en las zonas de estudio.

\begin{tabular}{clccc}
\hline Zonas & Localidad & $\begin{array}{c}\text { Biomasa } \\
\text { tonFV/ } \\
\text { ha/c }\end{array}$ & $\begin{array}{c}\text { Biomasa } \\
\text { tonMS/ } \\
\text { ha/c }\end{array}$ & $\begin{array}{c}\text { P. R. } \\
\text { días }\end{array}$ \\
\hline \multirow{2}{*}{$\begin{array}{c}\text { GUACHUCAL } \\
\text { Zona 1 }\end{array}$} & Arvela & 28,3 & 6,13 & 49 \\
& Cualapud & 23,1 & 4,8 & 51 \\
Sta. Rosa & 22 & 5,11 & 52 \\
\hline Promedio & & $\mathbf{2 4 , 4 7}$ & $\mathbf{5 , 3 5}$ & $\mathbf{5 0 , 6 7}$ \\
\hline \multirow{2}{*}{ IPIALES } & Los marcos & 12 & 1,66 & 41 \\
Zona 2 & San Luís & 15 & 2,07 & 40 \\
& Sta. bárbara & 12 & 1,66 & 38 \\
\hline Promedio & & $\mathbf{1 3}$ & $\mathbf{1 , 8 0}$ & $\mathbf{3 9 , 6 7}$ \\
\hline \multirow{2}{*}{ PAST0 } & Mocondino & 17,8 & 4,71 & 42,3 \\
Zona 3 & S. Fernando & 16,6 & 3,1 & 53 \\
& Pasto & 19,5 & 5,59 & 44 \\
\hline Promedio & & $\mathbf{1 7 , 9 7}$ & $\mathbf{4 , 4 7}$ & $\mathbf{4 6 , 4 3}$ \\
\hline
\end{tabular}

Variables bromatológicas. La Tabla 4 presenta los resultados observados en la composición nutricional en cada una de las zonas y sus localidades. El pasto Saboya presentó una calidad nutritiva aceptable, puesto que para la zona 1 , los valores promedio de proteína cruda (PC) y verdadera (PV) fueron de 14,55 y 11,27\% respectivamente. Para la zona 2, los valores promedio fueron de 17,88\% para proteína bruta y de 13,29\% para proteína verdadera. Para la zona 3 , los valores promedio de proteína bruta y verdadera fueron de 12,57 y 8,12\% respectivamente, como se puede observar en la Tabla 3. Bernal (2003) reporta un valor para proteína cruda de 11,95\% para esta gramínea en prefloración, por lo que se infiere que el nivel de proteína del forraje en las tres zonas es adecuado. En cuanto a los niveles de proteína verdadera, se puede observar que el nivel de Nitrógeno No Proteico (NNP) fue ligeramente mayor en el forraje recolectado en la zona 2 , dado que la cosecha se realizó a una edad temprana del pasto.

La zona 1, como lo muestra la Tabla 4, reportó un promedio de FDN de 55,59\%, así como un valor promedio para FDA de 31,74\%. En la zona 2 , los valores promedio para FDN y FDA fueron de 59,66 y $33,64 \%$ en su orden, mientras que la zona 3 reportó valores de FDN de 62,38\% y FDA de $36,97 \%$. Los valores obtenidos en las dos zonas para el componente FDN están dentro del rango establecido por Bernal (2003), entre $40 \mathrm{y}$ $80 \%$, esto a causa de la recolección del pasto a una edad temprana. Los valores reportados para FDA fueron bajos debido a la temprana edad al corte y condiciones favorables de fertilidad y precipitación en comparación al obtenido por Formoso (2012), quien afirma que 51,5\%, de fibra del pasto Saboya es alto y que un bajo nivel, especialmente de FDA, se traduce en un contenido alto de hemicelulosa, carbohidrato de mayor solubilidad $y$ fuente energética aprovechable. Los valores promedio de NDT fueron de 67,52, 55,67 y 54,86\% para las zonas 1, 2 y 3, quizás por la adecuada disponibilidad de nitrógeno en los suelos del ensayo. Dichos valores son bastante buenos si se tiene en cuenta lo afirmado por Kolver (2003), quien argumenta que una res para su mantenimiento requiere un $56 \%$ de NDT con base en la materia seca suministrada, lo cual indica que el pasto Saboya puede llenar estos requerimientos de manera satisfactoria, como lo describe la Tabla 4. 


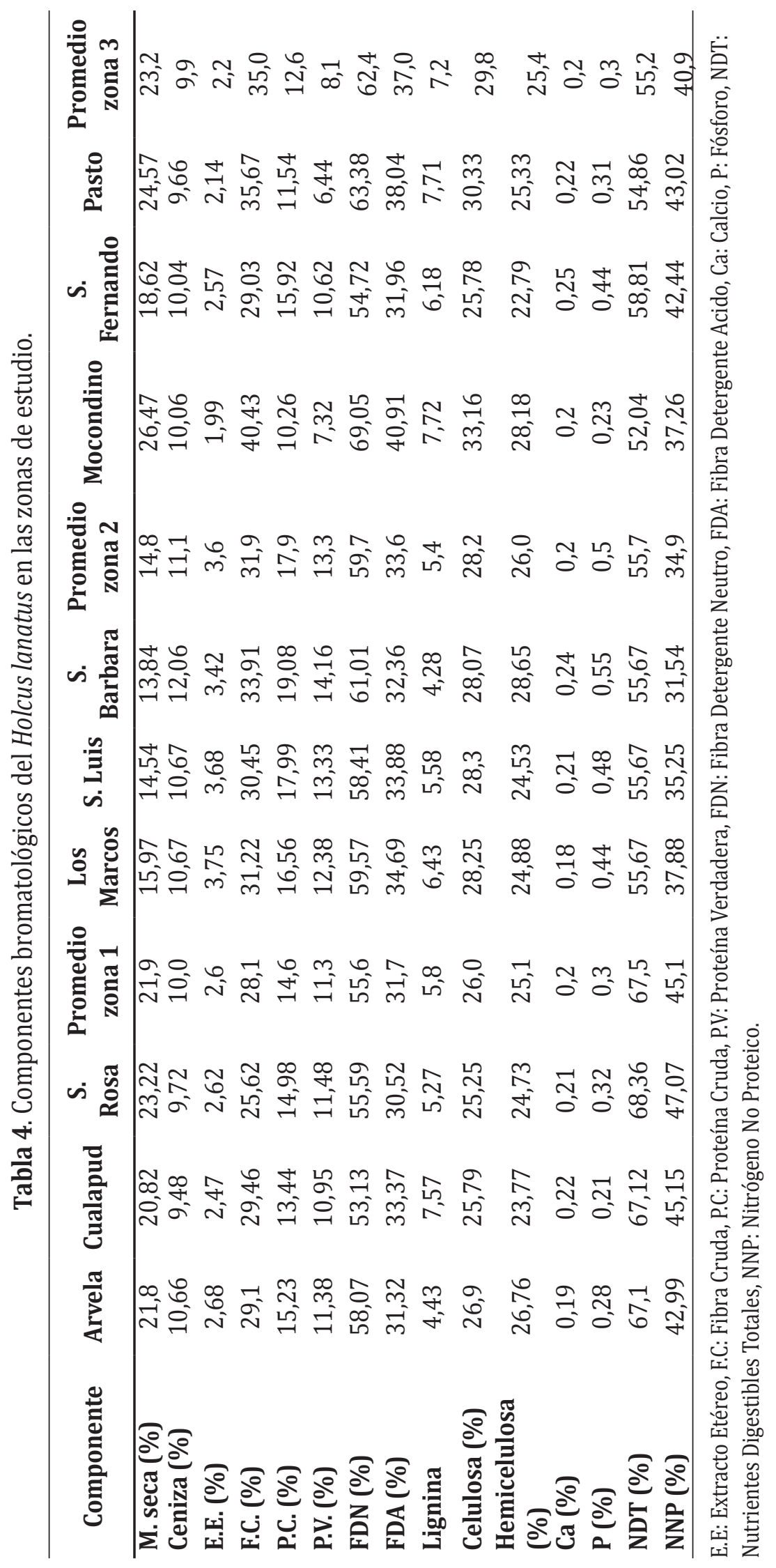




\section{Variables edáficas}

Variables Químicas: El pH de los suelos fue ácido en todas las zonas. La zona 1 obtuvo un $\mathrm{pH}$ de 5,34, en la zona 2 un pH de 5,6 y la zona 3 un pH de 5,25, resultados que confirman lo reportado por García (2003), quien afirma que el $H$. lanatus es una especie que prospera muy bien en suelos con pH entre 5 - 7,5 y en suelos más ácidos. En cuanto al porcentaje promedio de materia orgánica en el suelo, se obtuvieron valores de 13,52\% para la zona 1, 8,4\% para la zona 2 y $8,05 \%$ para la zona 3. Según Durán (2003), en climas fríos, los valores superiores al $10 \%$ de materia orgánica se consideran altos. La capacidad de intercambio catiónico promedio fue alta en las tres zonas, con 30,45meq/100g para la zona $1 ; 23,43 \mathrm{meq} / 100 \mathrm{~g}$ para la zona 2 y $27,54 \mathrm{meq} / 100 \mathrm{~g}$ para la zona 3 . Zapata (2004) cataloga una capacidad de intercambio catiónico por encima de 20meq/100g como alta. La elevada condición de esta variable está influenciada directamente por los contenidos medios de materia orgánica debido a la alta CIC, tal como lo corrobora Fajardo (2005).

Variables Físicas: La densidad aparente promedio fue similar en las tres zonas: 0,8, 0,9 y 0,91 para la zona 1,2 y 3 correspondientemente. Esto concuerda con Guerrero (1995), quien determinó que los suelos de Guachucal, Ipiales y Pasto presentan una densidad aparente por debajo de 1,0g/cc. La densidad estuvo íntimamente relacionada con los contenidos de materia orgánica presentes en las zonas de estudio; además, este valor bajo es indicativo de una compactación leve del suelo, lo que facilitó la penetración y desarrollo del sistema radicular de las plantas. El porcentaje promedio de porosidad para las zonas 1,2 y 3 , fue de $65,54,63,7$ y $60,7 \%$ respectivamente, lo que clasifica a los suelos de las tres zonas como de alta porosidad. Para Burbano (2014), ésta es una propiedad que favorece la retención, movimiento de agua, aireación, transferencia de oxígeno en el medio radical y la facilidad con que las raíces puedan anclar y sostenerse en el suelo.

La infiltración promedio en los suelos de las zonas 1 y 3 fue de 9,42cm/h y 7,24cm/h respectivamente, catalogadas como rápida, mientras que en la zona 2 la infiltración se catalogó como moderadamente lenta, con un valor promedio de $1,03 \mathrm{~cm} / \mathrm{h}$. Si bien lo deseable es que los suelos tengan mayor infiltración para su propia conservación, como lo afirma Fajardo (2005), se observa que éste no es factor limitante en el desarrollo satisfactorio del pasto Saboya. En cuanto a la penetrabilidad, se obtuvo un valor leve para la zona 1 , con $1,08 \mathrm{Mpa} / \mathrm{cm}^{2} \mathrm{y}$ un valor medio para las zonas 2 y 3 , con $1,8 \mathrm{Mpa} / \mathrm{cm}^{2}$ y $1,76 \mathrm{Mpa} / \mathrm{cm}^{2}$, respectivamente, lo que indica que, en general, en las diferentes zonas de estudio se presentó un nivel significativo de penetrabilidad, ya que en las tres zonas se supera el 0,59Mpa/ $\mathrm{cm}^{2}$, propuesto por Bernal (2003) como el nivel óptimo máximo de penetrabilidad; no obstante, el desarrollo radicular fue satisfactorio, obedeciendo a una buena cantidad de poros a causa de la baja densidad aparente encontrada.

\section{Análisis de Componentes Principales (ACP)}

El ACP agrupa las variables que están cerca o que son similares para formar una variable sintética con características propias en comparación con las demás, según Pérez (2004). En la zona 1 se obtuvo un total de seis componentes principales (CP), de los cuales tres explican el 67\% $(\mathrm{P}<0,05)$ del total de la variabilidad en el experimento. Para el caso de la zona 2, se establecieron cinco CP, de los cuales dos explican el 71\% $(\mathrm{P}<0,05)$ de la variabilidad total. Para la zona 3 se obtuvo un total de seis CP, de los cuales tres explican el $69,26 \%(\mathrm{P}<0,05)$ de la variabilidad.

\section{Zona 1, Guachucal.}

Componente 1: Producción de biomasa y calidad nutritiva. Este componente determinó que una capacidad de intercambio catiónico alto 
es favorable para obtener una mayor producción de biomasa y calidad nutritiva, debido a que el pasto está recibiendo adecuadamente los elementos necesarios de parte de la solución del suelo. Lo anterior está respaldado por Castro (1998), quien opina que en un suelo es deseable que se presente una CIC alta, asociada con una buena saturación de bases, ya que esto indica un gran potencial de suministro y reserva de nutrientes. La producción de forraje de calidad también se asocia con buenos contenidos de potasio, magnesio y calcio en el suelo. El estudio también reveló que los altos contenidos de azufre y hierro en los suelos perjudican el desarrollo de la gramínea, al igual que las intensidades lumínicas altas disminuyeron la calidad nutritiva del forraje. Bernal (2003) menciona que muchas especies de pastos no responden muy bien a densidades de luz mayores de $1 / 3$ de la máxima luz solar, de manera que sufren pérdida de agua debido a la apertura y cierre de los estomas al sufrir una excesiva exposición a la misma. La respuesta de las plantas a la luz no es lineal, es decir, al aumentar la intensidad lumínica, la fotosíntesis no aumenta proporcionalmente (Figura 1).
Componente 2. Contenido de azufre en el pasto. Teniendo en cuenta los resultados presentados en la Figura 2, en este componente se determinó que la cantidad de azufre en el forraje se vio favorecida por el incremento del pH y la presencia de manganeso en cantidades aprovechables por la planta y una infiltración moderada. Según Guzmán (2004), el azufre es asimilable a $\mathrm{pH}$ ácido, por esta razón, el pasto Saboya está muy bien adaptado a pH ácidos, con alta capacidad de intercambio catiónico, debido al contenido de materia orgánica en los suelos. Castro (1998) menciona que los suelos con $\mathrm{pH}$ alto son deficientes en manganeso, es por esto que el nivel de $\mathrm{pH}$ debe mantenerse en un rango óptimo de acidez en el que las concentraciones de manganeso no sean tan bajas que haya deficiencia, ni tan altas que se presente toxicidad. La participación de la infiltración en este componente ocurre de manera inversa, siendo deseable, para un óptimo aprovechamiento de minerales, que se mantengan valores medios de infiltración, de tal manera que haya un movimiento adecuado de agua en el suelo para una movilización óptima de nutrientes.

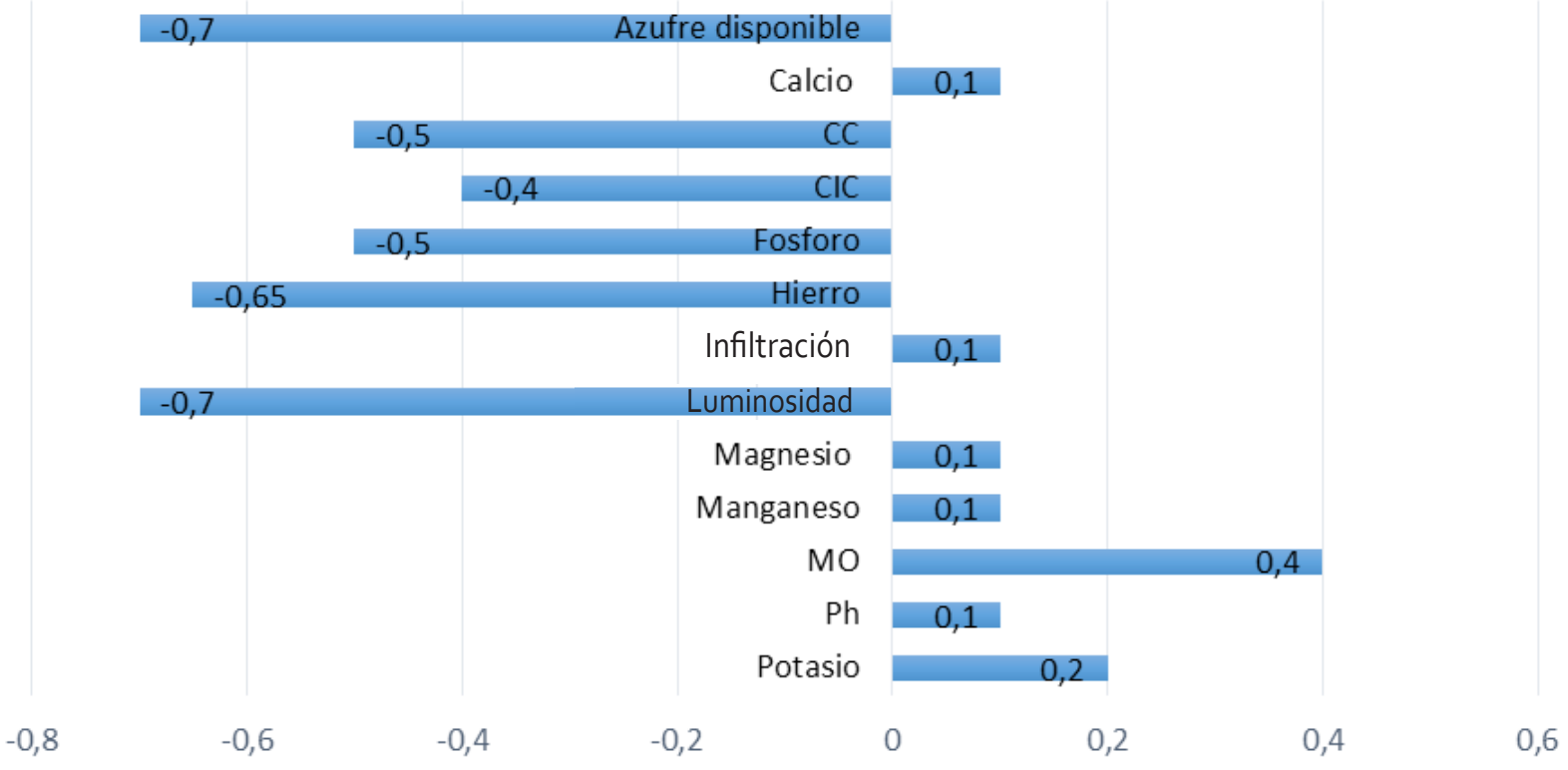

Figura 1. Aporte de las variables edáficas incidentes en la producción de biomasa del pasto Saboya. 


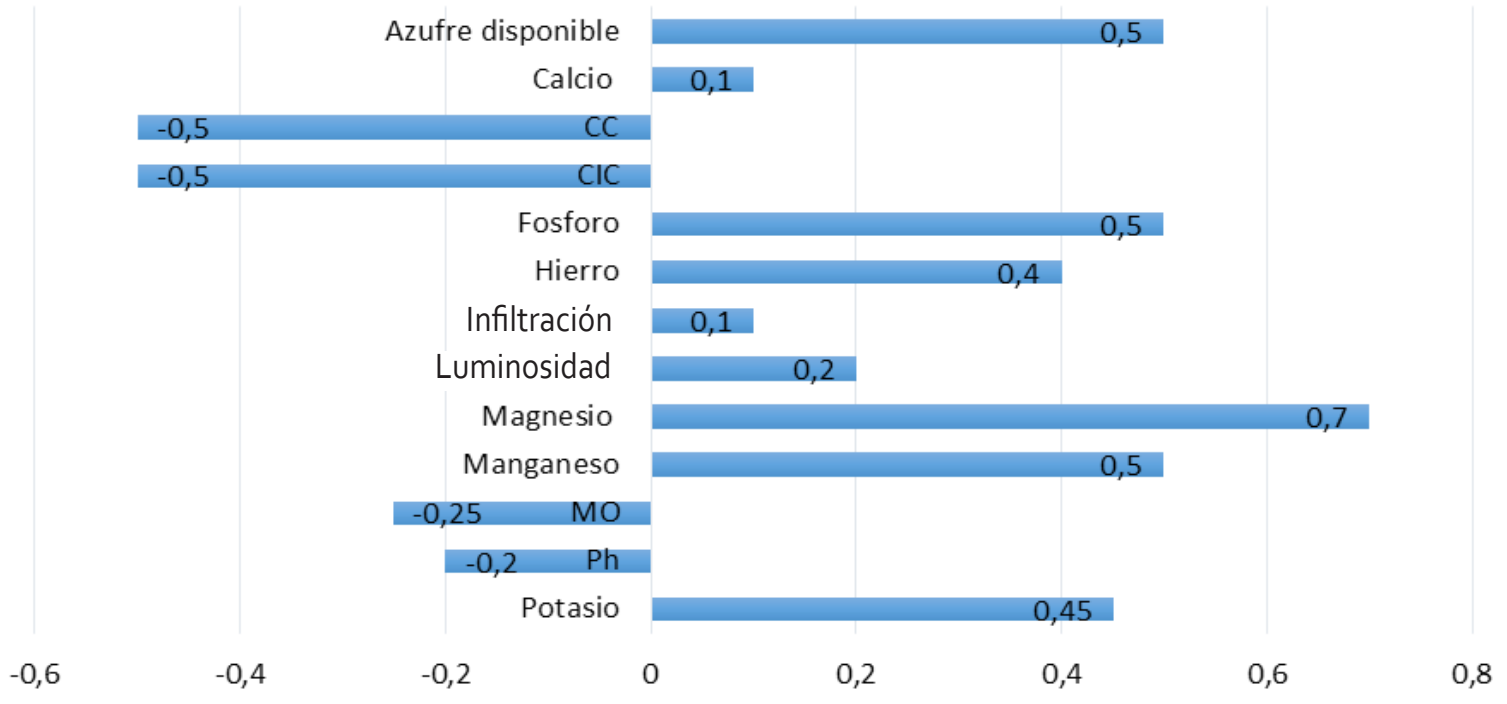

Figura 2. Aporte de las variables edáficas incidentes en el desarrollo de los forrajes.

Componente 3: Contenido de lignina. El contenido de lignina será menor en la medida en que aumente el contenido de materia orgánica, de potasio en el suelo y cuando disminuya la capacidad de campo del suelo. Al respecto, Guzmán (2004) menciona que la materia orgánica del suelo es una fuente de nitrógeno, fósforo y azufre, útiles para la nutrición de las plantas, lo cual significa que en los suelos estudiados la materia orgánica propicia la disponibilidad de elementos importantes utilizados por las plantas para su crecimiento y desarrollo, por la alta capacidad de intercambio catiónico que presenta. Entre estos elementos se debe resaltar al nitrógeno, puesto que, según Castro (1998), promueve el rápido crecimiento, y cuando su contenido es muy alto, retarda la maduración. De esto se concluye que el forraje que crece donde hay altos contenidos de materia orgánica se verá más precoz y la maduración tardará un poco más en comparación con los suelos pobres. El potasio, participa en la síntesis de carbohidratos solubles en la planta, por lo tanto, mientras las concentraciones de potasio sean altas, el contenido de lignina será bajo en el forraje. Respecto a la capacidad de campo, se obtuvo una relación inversamente proporcional con el contenido de lignina, ya que los suelos utilizados en el estudio tuvieron texturas franco - arcillosas, lo que facilita la retención de agua (Figura 3).

\section{Zona 2, Ipiales.}

Componente 1. Producción de biomasa. El contenido de lignina se relacionó con una alta producción de biomasa, con una influencia directa del $\mathrm{pH}$, calcio, fósforo y magnesio y de forma indirecta por la materia orgánica e infiltración. El comportamiento productivo de esta gramínea se da en un pH moderadamente ácido y puede incrementar su producción al acercarse a la neutralidad, ya que en esta condición se da una mayor disponibilidad y asimilación de minerales que son necesarios para el crecimiento de esta planta. Al respecto, Bernal (2003) afirma que el mejor rango de $\mathrm{pH}$ para el crecimiento de las plantas se encuentra entre 5,5 y 6,5. Los niveles de calcio en el suelo tuvieron una relación directa con el volumen de producción de biomasa, ya que, según García et al. (2012) el calcio estimula el desarrollo de las raíces y hojas. La relación directa del fósforo con la producción de biomasa se dio por su importancia para la planta, ya que para Moreno (2015) el fósforo desempeña un importante papel metabólico en la respiración, fotosíntesis y transferencia de energía, es determinante para el desarrollo de raíces y de los tejidos meristemáticos. 


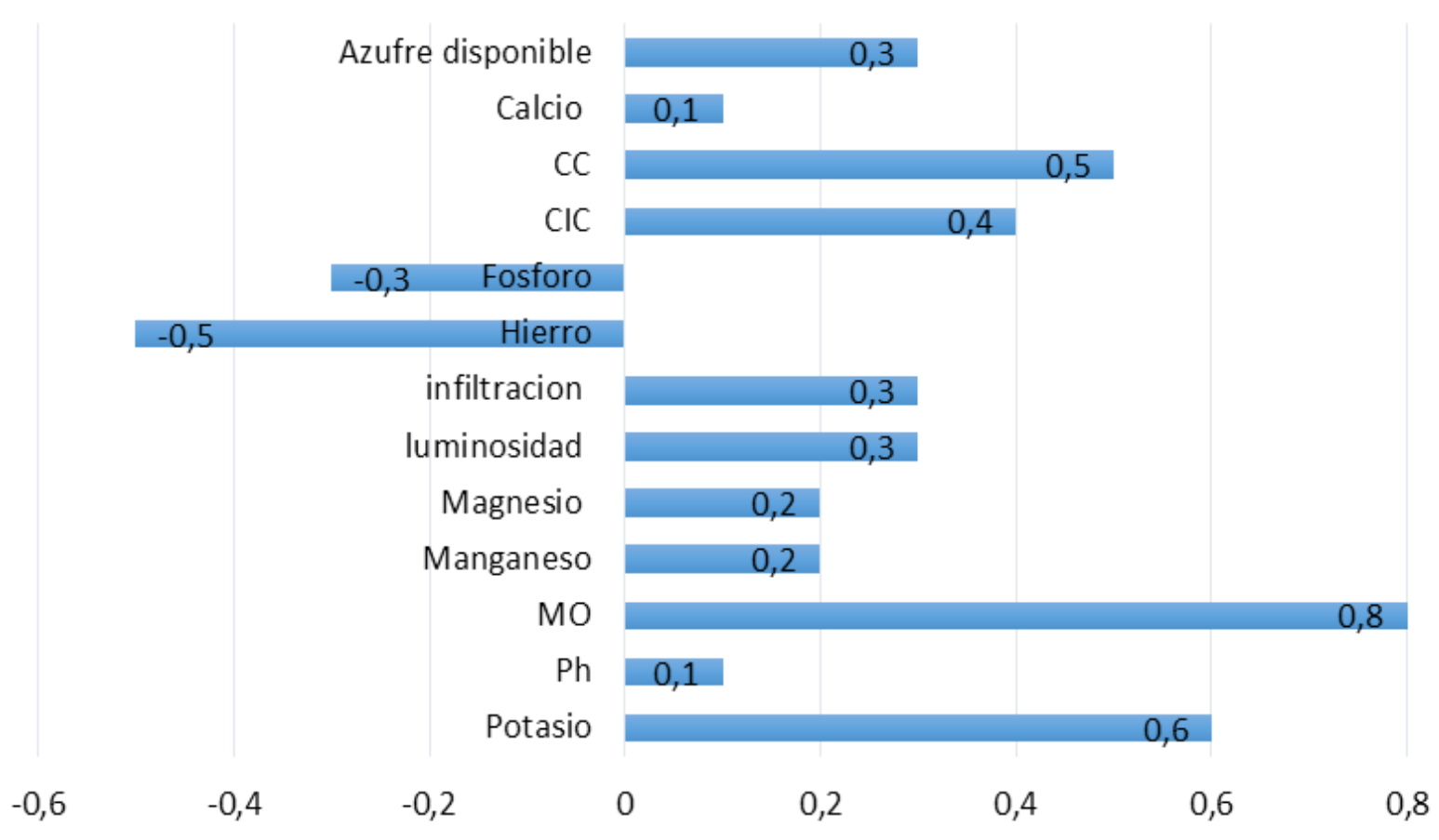

Figura 3. Aporte de las variables edáficas incidentes en el contenido de lignina.

Si bien la relación de los niveles de calcio y fósforo ejercieron influencia directa en el volumen de producción de biomasa, ésta no fue estadísticamente significativa $(\mathrm{P}<0.05)$, como se pudo observar también que los niveles elevados de magnesio influyen positiva y significativamente en el volumen de producción forrajera de este pasto. Al respecto, Moreno (2015) argumenta que este elemento hace parte de la clorofila, activa varios sistemas enzimáticos en las rutas metabólicas de la planta involucrando la respiración, fotosíntesis y transformación de lípidos e intervienen en el transporte del fósforo, por lo que con niveles adecuados de magnesio se produce un mayor crecimiento en la planta. En lo referente al contenido de materia orgánica, se puede decir que se encontró un efecto similar al reportado en la zona 1, contribuyendo al crecimiento vegetal mediante su efecto sobre propiedades físicas, al estabilizar y mejorar la estructura y capacidad de retención de humedad y en las propiedades químicas al aumentar la capacidad de intercambio catiónico y regular el pH. De esta manera, sirve como reservorio de nutrientes y mejora las condiciones para el crecimiento microbiano. Algo parecido sucedió con la variable infiltración, que mostró un comportamiento similar al de la zona 1 al tener un efecto negativo en la medida en que éste se incrementa, tal como describe en la Figura 4. 


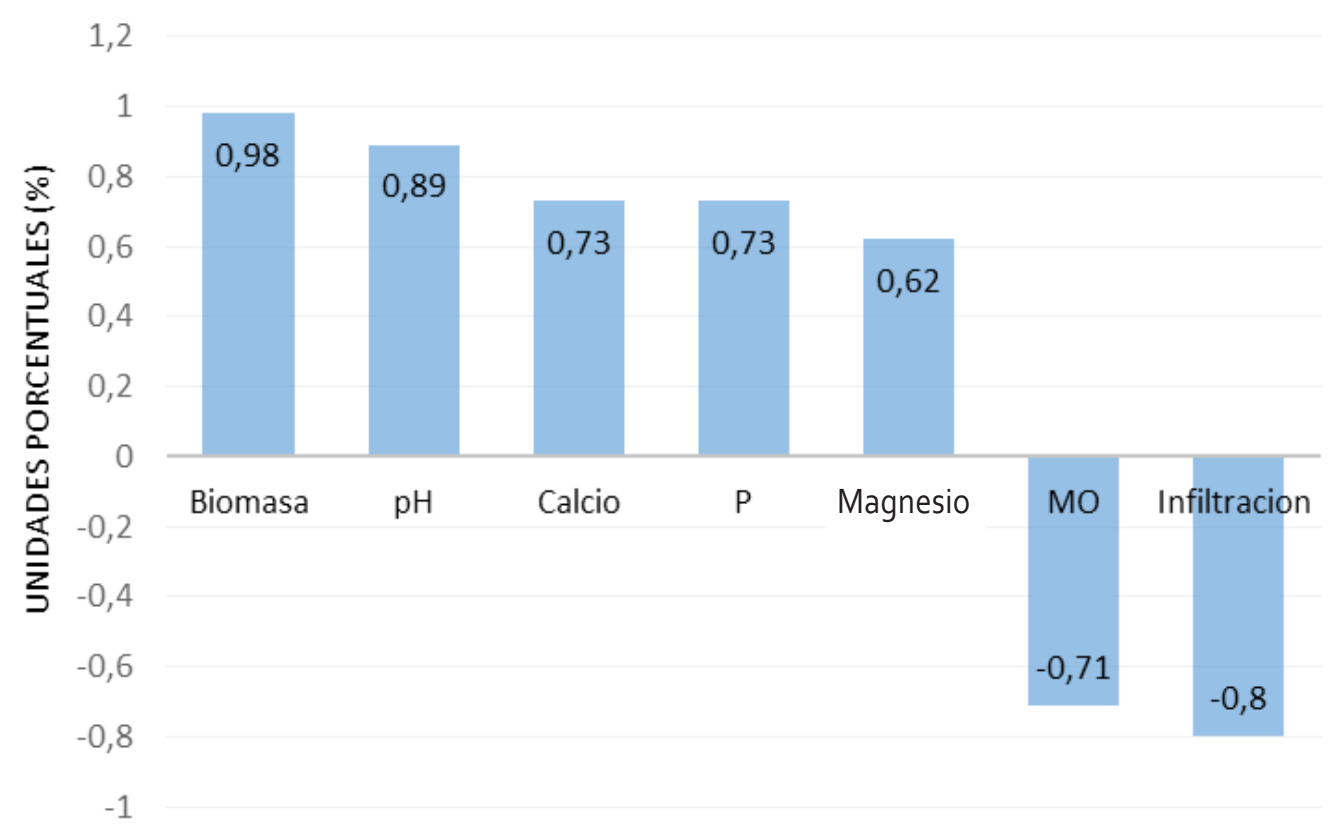

Figura 4. Correlación de las variables estudiadas con el componente principal uno.

\section{Componente 2. Propiedades químicas} y físicas del suelo. En este componente, participan de manera directa la capacidad de campo y la capacidad de intercambio catiónico e indirectamente el azufre y la penetrabilidad. Si bien la capacidad de campo ejerció un efecto directo sobre la producción de biomasa, éste no fue significativo, por lo que se puede decir que la capacidad de campo encontrada en las zonas de estudio, la cual osciló entre 43,77 y 49,4\%, permitió que haya cantidad de oxigeno suficiente y agua retenida con poca fuerza por el suelo; además, existióunabuena capacidad de retención de agua y aireación del suelo, que incrementó las posibilidades de la planta para obtener los nutrientes necesarios para su crecimiento. La misma situación se presentó con el componente capacidad de intercambio catiónico, el cual tuvo una influencia positiva, pero no significativa en el volumen de biomasa. Los niveles de capacidad de intercambio catiónico en las zonas de estudio fueron altos y estuvieron asociados a los altos contenidos de materia orgánica, característicos del clima frío.
En este sentido, Bernal (2003) afirma que a mayor capacidad de intercambio catiónico, mayor potencial de fertilidad del suelo. Esta capacidad depende del contenido de materia orgánica y el pH del suelo. El azufre también tuvo una influencia directa, pero no significativa en la producción de biomasa. Cabe resaltar que los requerimientos de este nutriente por parte del pasto, no son mayores que los disponibles en el suelo en todos los sitios estudiados. La resistencia a la penetración (penetrabilidad) no tuvo efecto sobre la producción de biomasa. Esto obedeció a que el sistema radicular del pasto Saboya no mostró tendencia a profundizar las capas el suelo, como se representa en la, se debe considerar que la especie estudiada es una gramínea cuya profundidad radicular no supera $10520 \mathrm{~cm}$ de la capa arable del suelo, por lo que su relación con la penetración dentro de las capas as profundas del suelo, no resulto considerable como lo muestra la Figura 5; lo que sugiere que dentro de los arreglos silvopastoriles se debe considerar otras especies de Pastos cuya profundidad radicular sea mayor y así poder contribuir a esta variable. 


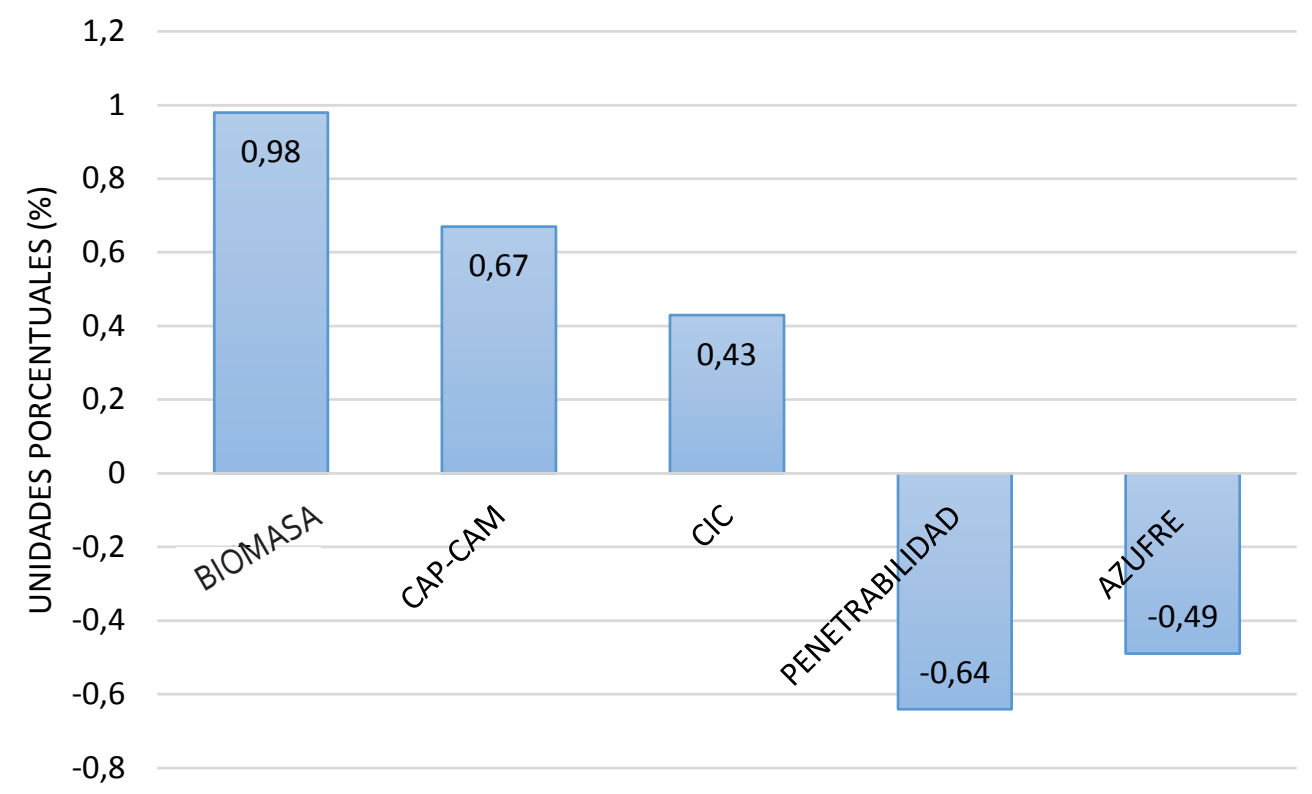

Figura 5. Correlación de las variables estudiadas con el componente principal dos.

\section{Zona 3. Pasto.}

Componente 1. Periodo de recuperación y producción de biomasa. Este componente representa un largo periodo de recuperación y producción de biomasa, que se ve influenciado inversamente por el $\mathrm{pH}$ y el potasio del suelo. En cuanto a los minerales del suelo, el boro influye de manera directa; básicamente se debe al efecto que tiene el $\mathrm{pH}$ sobre estos elementos del suelo. Cabe destacar que se aprecia una relación muy estrecha y directa entre el periodo de recuperación y el contenido de proteína verdadera. Al respecto, Guzmán (2004) menciona que la disponibilidad máxima de los nutrientes primarios es mayor a un valor de $\mathrm{pH}$ cercano a la neutralidad, lo cual repercute en el contenido de minerales en el pasto. De acuerdo con Alarcón (2001), la deficiencia de boro generalmente detiene el crecimiento de la planta, primero dejan de crecer los tejidos apicales y las hojas más jóvenes. Esto indica que el boro no se transloca fácilmente en la planta, como se observa en los valores correspondientes en la Figura 6, se debe considerar que el boro es un elemento poco móvil dentro de las estructuras vegetales de absorción de nutrientes, por lo que este comportamiento sugiere mayor énfasis al momento de medir estas variables y de determinar sus resultados.
Componente 2. Calcio del forraje. En este componente sobresale la variable Calcio del forraje, el cual se encuentra influenciado directamente por la materia orgánica (MO) y temperatura, y de manera indirecta con la velocidad de infiltración y la precipitación. Lo que quiere decir que si se aumenta el contenido de MO y temperatura, se elevarán los contenidos de calcio en el forraje. Para Guzmán (2004), la materia orgánica, igual que la arcilla, puede retener por medio de la absorción altas cantidades de nutrimentos en forma iónica. Por otro lado, Lora (2001) afirma que la absorción de nutrimentos está relacionada con la actividad metabólica, la cual, a su vez, es dependiente de la temperatura, tal como lo presenta la Figura 7; cuando se trata sobre la movilidad de nutrientes de debe considerar todos los factores a que ellos están asociados puesto que existen relaciones sinérgicas y antagónicas entre algunos elementos debido a su valencia y a su movilidad no solo dentro del suelo, sino también una vez estén dentro de la planta, todos estos factores de absorción, movilidad y translocación se deben evaluar desde el punto de vista químico pero considerando las variables climáticas, como temperatura, humedad, etc. ya que estas afectan el sistema de absorción por parte de las plantas. 


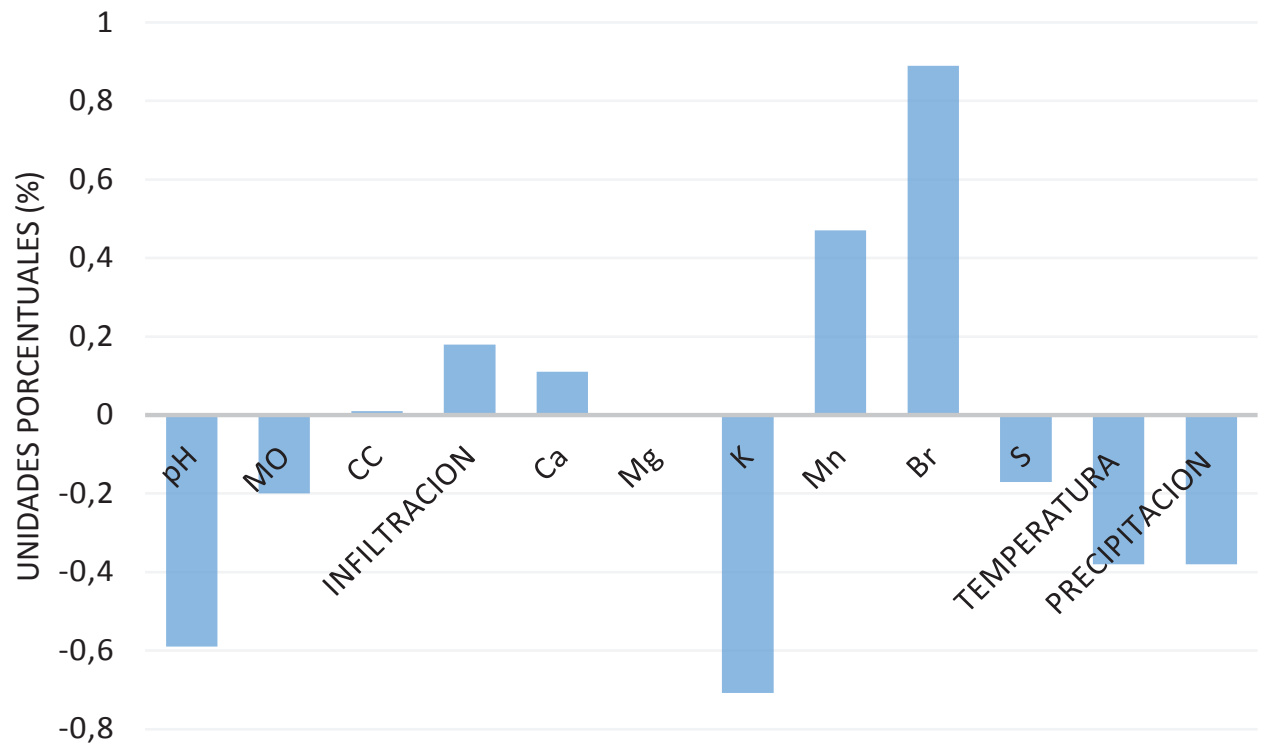

Figura 6. Grado de variación de las variables del componente 1.

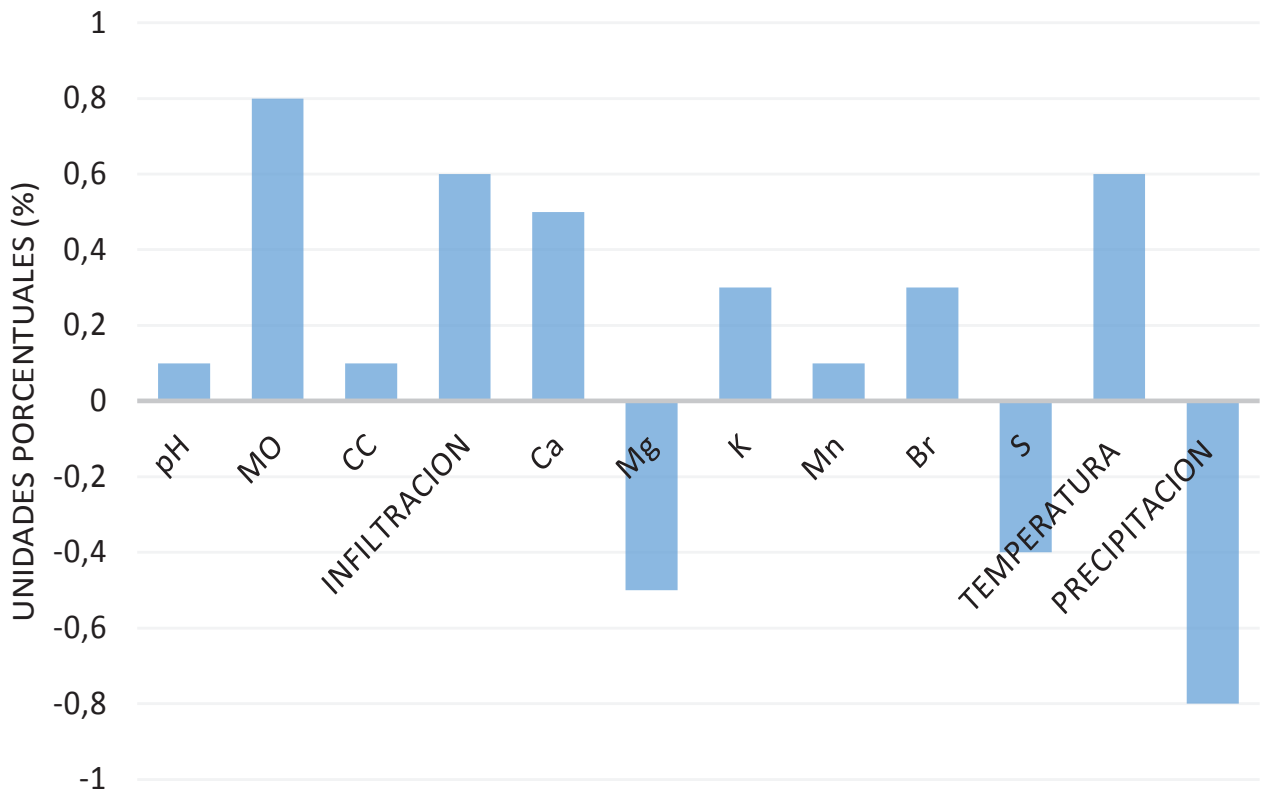

Figura 7. Variación de las variables del componente dos.

Componente 3. Carbohidratos estructurales. En este componente se identifican los carbohidratos estructurales ya que las variables quesobresalieronfueronceniza,ligninayextracto etéreo, lo cual se ve afectado directamente por los minerales calcio y manganeso del suelo, lo que significa que para obtener una mejor calidad del pasto Saboya se necesita tener bajos valores de calcio y manganeso en el suelo. De acuerdo con Guzmán (2004), todas las plantas absorben los minerales del suelo y los sintetizan en compuestos orgánicos complejos de los cuales están formados sus tejidos. 
Guzmán (2004) sostiene que el calcio, por ser un nutriente de naturaleza estructural, constituye un componente de paredes y membranas celulares y debe estar presente para la formación de nuevas células. El calcio, una vez que se deposita en los tejidos vegetales, no es posible removerlo, por lo que los tejidos jóvenes son los primeros en ser afectados cuando existen deficiencias de este nutriente Berlijn (2010). Afirma que el manganeso interviene en la síntesis de proteína. Está involucrado en diversos sistemas de oxidaciónreducción dentro de la planta y es esencial en los procesos de la respiración y el metabolismo del nitrógeno y los azucares. El manganeso es inmóvil dentro de la planta y se absorbe del suelo como $\mathrm{Mn}^{++}$y $\mathrm{Mn}^{+++}$, como se observa en la Figura 8, se puede observar que la mayoría de las variables presentan cambios considerables en el contenido de micro elementos sin embargo, también se puede determinar que las variables climáticas presentan una correlación negativa lo que sugiere que estas no influyen de manera directa dentro de este componente.

\section{CONCLUSIONES}

La producción de biomasa en forraje verde presentó una relación directa con la capacidad de intercambio catiónico e inversa con el azufre disponible, el hierro y la luminosidad. El pasto Saboya no requiere de una radiación solar directa y prospera en condiciones de baja luminosidad. Presentó una producción y calidad nutritiva aceptables en suelos con reacciones ácidas.

La cantidad alta de fauna edáfica estuvo favorecida por la humedad, temperatura y disponibilidad de materia orgánica en el suelo. La presencia dela subclase Oligochaeta (lombriz de tierra) estuvo relacionada con los contenidos medios de MO, el pH del suelo y el grado textural.

Conflicto de intereses: Los autores declaran que no hay conflicto de interés.

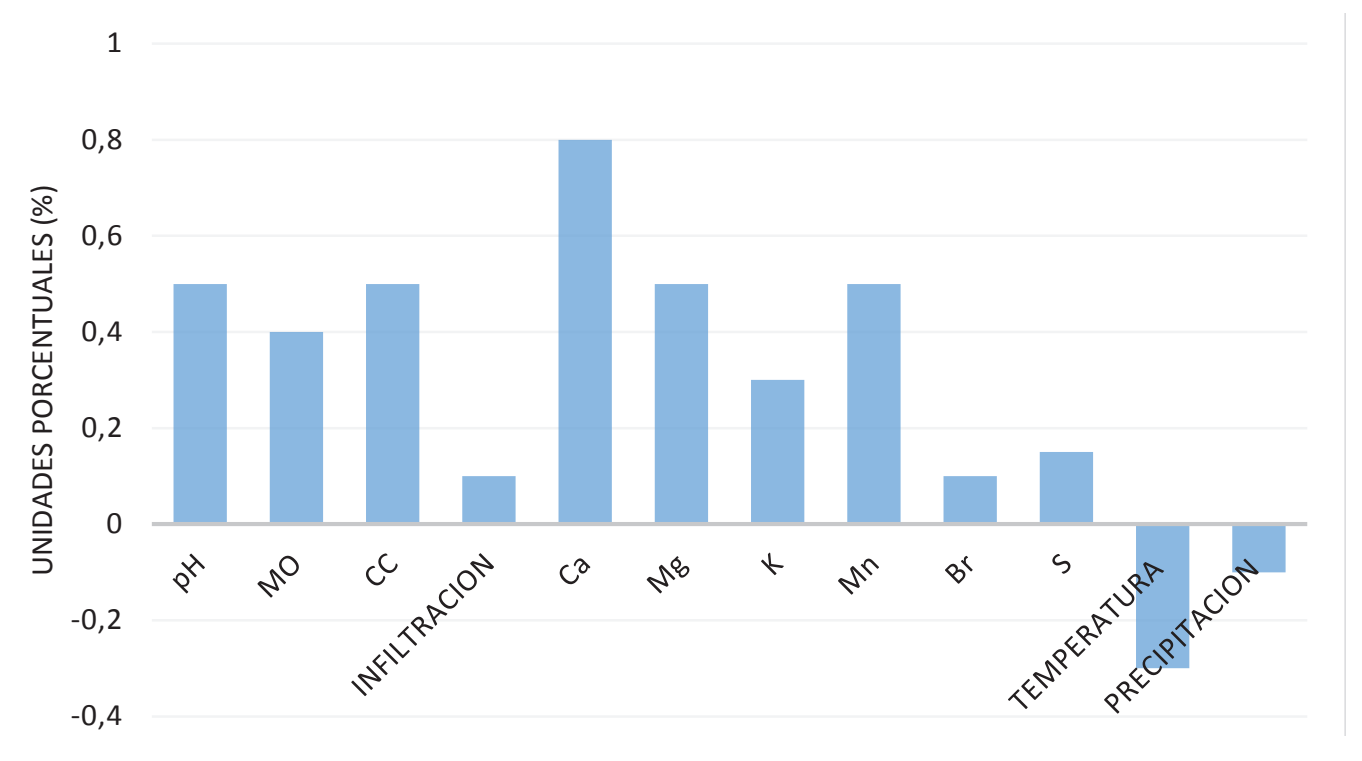

Figura 8. Variación de las variables del componente tres. 


\section{REFERENCIAS BIBLIOGRÁFICAS}

Alarcón, A. (2001). El boro como nutriente esencial. Horticultura. 155: 36-46.

Apráez, E. (1994). Análisis químico de los alimentos. Pasto: Editorial Universidad de Nariño. 167p.

Begon, M., Harper, J. \& Towsend, C. (1999). Ecología, individuos, poblaciones y comunidades. $3^{\mathrm{a}} \mathrm{ed}$. Oxford, E.E.U.U: Blackwell Science Ltd. 1148p.

Berlijn, J. (2010). Cultivos Forrajeros. Manual para Educación Agropecuaria. México D.C: Editorial Trillas. $112 \mathrm{p}$.

Bernal, J. (2003). Pastos y forrajes tropicales. $4^{\circ} \mathrm{ed}$. Bogotá: Ángel agro-ideagro. 550p.

Burbano, H. (2014). Eeducación para la sostenibilidad. Suelos Ecuatoriales. 44 (2): 138-150.

Cabrera, A. (2007). Plan de Desarrollo del municipio de Aldana periodo 2004-2007. Aldana: Alcaldía Municipal de Aldana. 6p.

Castro, H. (1998). Fundamentos para el Conocimiento y Manejo de Suelos Agrícolas. Tunja: Instituto Universitario Juan de Castellanos. 115p.

Durán, F. (2003). Volvamos al Campo: Manual de Cultivos Orgánicos y Alelopatía. Bogotá: Grupo Latino. 74p.

Enríquez Quiroz, J.F. \& Quero Carrillo, A.R. (2006). Producción de semillas de gramíneasy leguminosas tropicales. México: INIFAP. 125p.

Fajardo, G. (2005). Evaluación preliminar de algunas variables edafoclimáticas y productivas del sistema agroforestal con Quillotocto (Tecota stans) (L) H.B.K, Acacia amarilla, (Acacia decurrens) Hill y Kik, (pennisetum clandestimun) (Hochst) en la granja de Botana. Recuperado de https:// es.slideshare.net/dirseo/establecimiento-y-evaluacion-preliminar-de-los-arreglos-de-1.

Formoso, D. (2012). Efecto de la fertilización (macro y micronutrientes) en la producción de semillas de gramíneas forrajeras tropicales. Recuperado de http://www.pasturasdeamerica. com/articulos-interes/notas-tecnicas/efectofertilizacion-produccion-semillas-gramineasforrajeras/.
García, Y., Ramírez, W. \& Sánchez, S. (2012). Indicadores de la calidad de los suelos: una nueva manera de evaluar este recurso. Pastos y Forrajes. 35 (2):125-138.

García, J. (2003). Crecimiento y calidad de gramíneas forrajeras en la Estanzuela. Serie técnica No.133. Montevideo, Uruguay: Unidad de Agronegocios y Difusión del INIA. 140p.

Guerrero, R. (1998). Fertilización de cultivos en clima frío. Segunda Edición. Honduras: Monómeros. 370p.

Guzmán, M. (2004). Manual de Fertilizantes para Cultivos de Alto Rendimiento. México: Limusa. $60 \mathrm{p}$.

Heredia, E. (2015). Densidad real y aparente y porosidad del suelo. Lima: Pontificia Universidad Católica del Perú. Facultad de Ciencias e Ingeniería. 35p.

Horwitz, W. (2005). Official Methods of Analysis of AOAC International 18th. Connecticut, United States of America. U.S.A government: AOAC. 45p.

IDEAM - Instituto de Hidrología, Meteorología y Estudios Ambientales. (2017). Tiempo y clima. Recuperado de http://www.ideam.gov.co/web/ tiempo-y-clima/climatologico-mensual?p_p_ $\mathrm{id}=110 \_$INSTANCE_xYvlPc4uxk1Y\&p_p_ lif e cy cle $=0 \& p_{-} p_{-}$state $=$normal $\& p_{-} p_{-}$ mode $=$ view\&p_p_col_id $=$ column $-1 \&$ p_p_col_ count $=2 \& \_110 \_$INSTANCE_xYvlPc4uxk1Y_ struts_action $=\% 2 \mathrm{Fdocument}$ library_ display\%2Fview_file_entry\&_110_INSTANCE_ xYvlPc4uxk1Y_redirect=http $\% 3 \mathrm{~A} \% 2 \mathrm{~F} \% 2 \mathrm{Fwww}$. i d e a m.gov.co \% $2 \mathrm{Fw}$ eb \% $2 \mathrm{Fti}$ e m p o-y -

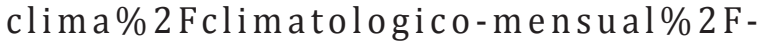
$\% 2$ Fdocument_library_display $\% 2 \mathrm{FxY}$ vlPc 4uxk1Y\%2 Fview\%2F $18512937 \%$ 3F_110_INSTANCE_xYvlPc4uxk1Y_redir ect $\% 3$ Dhttp $\% 253$ A $\% 252$ F $\% 252$ Fwww. ideam.gov.co\%252 Fweb\%252 Ftiempoy-cli ma \% $252 \mathrm{Fcli}$ matologi comensual\%253 F p_p_id \%253 D 110 _ INSTANCE_xYvlPc 4 uxk 1 Y \% 2526 p_p_ lifec y c l e \% 253 D $0 \% 2526 \mathrm{p}_{\text {_ }} \mathrm{p}_{\text {_ }}$ state\%253 D normal\%2526 p p mode \% $253 \mathrm{Dv}$ i e w \% $2526 \mathrm{p}_{\text {_ }} \mathrm{p}_{-} \mathrm{col}_{\text {_ }}$ id \% 253 D column-1\%2526 p_p_col_ count\%253D2\&_110_INSTANCE_xYvlPc4uxk1Y_ fileEntryId $=68186681$ 
Kolver, E. (2003). Nutritional limitations to increased production on pasture based systems. Proc. Nutr. Soc. 62: 291-300.

Lora, R. (2001). Factores queafectan la disponibilidad de nutrimentos para las plantas. En: Clavijo, J., Lora, R., Malavolta, E., Zapata, R., Espinosa, J., Amézquita, E., León, A., Guerrero, R., Muñoz, R., Burbano, H., Sánchez, L., Villar, H., Barrera, L. \& García, A. Fertilidad de suelos, diagnóstico y control. pp 41 - 42. 2ed. Bogotá: Sociedad Colombiana de la ciencia del suelo. 151p.

Moreno, A. (2015). Relación suelo-agua-plantas. Conceptos básicos. España: Escuela Universitaria de Ingeniería Técnica agrícola de Ciudad Real. $46 \mathrm{p}$.

Pérez, C. (2004). Técnicas de Análisis Multivariante de Datos. Aplicaciones con SPSS. Madrid: Pearson Education. 665p.

Zapata, R. H. (2004). Química de la acidez del suelo. Medellín, Colombia: Universidad Nacional de Colombia. 33p. 